\title{
Visuelle Vorgänger - Fabriken in der Malerei
}

Die ersten Fotografien russischer Industrieanlagen entstanden nicht in einem bildleeren Raum. Die fotografische Technik, die Lichtempfindlichkeit der Chemikalien und die Wetterverhältnisse bestimmten maßgeblich, welche Motive die Fotografen aufnehmen konnten. Neben diesen äußeren Rahmenbedingungen hatten Berufserfahrung und kulturelle Sehgewohnheiten die Blicke der Lichtbildner und ihr Gespür für Ästhetik zusätzlich geschult. Die Betrachtung und Entschlüsselung eines Bildes basiert folglich auf seiner kulturellen Kodierung sowie der Erfahrung des Betrachters. ${ }^{1}$ Darüber hinaus bestimmten zahlreiche Parameter die Herstellung einer Fotografie. Gerade in den ersten Jahrzehnten nach der Entwicklung der fotografischen Technik waren viele Personen, die das neue Gewerbe ergriffen, ausgebildete Maler. ${ }^{2}$ Entsprechend wirkmächtig waren Darstellungen von Industrieanlagen und Fabriken in der Bildenden Kunst für die Ausbildung einer fotografischen Bildsprache.

Zunächst waren insbesondere Gemälde aus West- und Nordeuropa Vorbilder für die Darstellungsweise der neuen Errungenschaften und Technik. Ausschlaggebend war, dass in Westeuropa bereits im 18. Jahrhundert industrielle Anlagen entstanden waren und die Künstler zu neuen Bildern und einer neuen Bildsprache inspiriert hatten. Im Zarenreich entwickelte sich die Industrie deutlich später und schlug sich überdies eher selten in der Malerei nieder. Dies bedeutete aber nicht, dass russische Künstler keine Industriedarstellungen kannten; nationale Grenzen spielten in der Kunstszene eine vergleichsweise geringe Rolle. In russischen Künstlerkreisen war es üblich, Teile der Ausbildung in Westeuropa zu absolvieren oder Reisen in die dortigen kulturellen Zentren zu unternehmen. Die Künstler hatten jedoch nicht nur auf ihren Reisen Gelegenheit Industriegemälde zu betrachten, Werke aus dem Ausland fanden auch ihren Weg ins Zarenreich, wenn beispielweise russische Käufer Industriebilder in Großbritannien erstanden und nach Russland brachten. ${ }^{3}$

1 Gustav Frank; Barbara Lange: Einführung in die Bildwissenschaft. Bilder in der visuellen Kultur, Darmstadt 2010, S. 21.

2 Elliot: The Photograph in Russia, S. 12; Saburova: Early Masters of Russian Photography, S. $38-39$.

3 Der spätere russische Konsul Aleksandr Bakster kaufte 1774/75 vom Englischen Künstler Joseph Wright of Derby (1734-1797) das Gemälde eines Dampfhammers für die Zarin Kathraina II. Christine Hoffmeister: Europäische Industriegemälde zwischen Rokoko und 
Weiter veröffentlichten russische Illustrierte im 19. Jahrhundert Stiche von Industrieanlagen und Industriegemälden aus anderen europäischen Ländern. ${ }^{4}$ So kannten die Maler im Zarenreich auch das monumentale „Eisenwalzwerk“ von Adolph Menzel (1815-1905), das in den Jahren 1872 bis 1875 entstand und 1875 in der Berliner Nationalgalerie ausgestellt war. ${ }^{5}$

Aufgrund dieser stilbildenden Funktion der westeuropäischen Industriemalerei wird im Folgenden ein kurzer Überblick gegeben, wie sich dieses Genre in Europa entwickelte, speziell die Darstellung von Fabriken. Er soll den Kontext skizzieren, in dem die ersten Fotografien entstanden, und zeigen, welche Bildsprache zu dieser Zeit üblich war. Leider gibt es bislang keine vertiefenden Forschungen zur vorrevolutionären Industriemalerei in Russland. In der Sowjetunion war das Interesse an Industriegemälden zwar prinzipiell vorhanden, Kunsthistoriker sahen aus ideologischen Gründen jedoch davon $\mathrm{ab}$, zu zeigen, dass das Zarenreich punktuell bereits einen hohen Grad an Industrialisierung erreicht hatte. Auch in den vergangenen $25 \mathrm{Jahren}$ etablierte sich die Industriemalerei nicht als lohnender Untersuchungsgegenstand der russischen Kunstgeschichte.

Malerei und Fotografie existierten nach 1839 auch im Zarenreich nebeneinander her und beeinflussten sich gegenseitig, weswegen die folgenden Ausführungen nicht mit der Entdeckung des fotografischen Verfahrens enden, sondern bis Ende des 19. Jahrhunderts reichen und anschließend einen kurzen Ausblick in das 20. Jahrhundert vornehmen.

\subsection{Faszinierendes Schauspiel und Orte außerhalb der Nation}

Industriebilder können eine Vielzahl an Motiven umfassen wie Förderund Fertigungsanlagen oder Verkehrsmittel und Bauwerke. Viele dieser Konstruktionen machten erst technische Neuentwicklungen möglich, so waren stählerne Eisenbahnbücken ein neues Element in der Natur. ${ }^{6}$ Bei der

Romantik, in: Klaus Türk (Hrsg.): Arbeit und Industrie in der bildenden Kunst. Beiträge eines interdisziplinären Symposiums, Stuttgart 1997, S. 27-39, S. 29.

4 Beispielsweise veröffentlichte die Vsemirnaja Illjustracija am 17. Februar 1873 einen Stich, der die Arbeit eines Dampfhammers in Woolwich, einem Stadtteil von London, zeigte.

5 http://germanhistorydocs.ghi-dc.org/sub_image.cfm?image_id=1312\&language=german (zuletzt eingesehen am 03.09.15). Eine Reproduktion des Gemäldes druckte 1900 auch die Illustrierte Niva ab. Das verdeutlicht, dass das Bild im Zarenreich bekannt war und die Herausgeber um die Jahrhundertwende davon ausgingen, dass es den Geschmack einer breiten Leserschaft ansprechen könne.

6 Francis Donald Klingender: Kunst und industrielle Revolution, Dresden 1974, S. 25; Jochen Luckhardt: Zum Verhältnis von Landschaft und Industrie in westfälischen Ansichten 
Darstellung von Fabriken existierten insbesondere zwei Traditionslinien, von denen ausgehend sich die Künstler der Thematik näherten: die Abbildung des Arbeitsprozesses und die Landschaftsmalerei. ${ }^{7}$ Generell ist bei Gemälden von Arbeitsabläufen und speziell von Industrieanlagen immer zu beachten, dass es sich bei den Bildern um den Blick von Laien auf das neue Phänomen der Industrialisierung handelte. Ingenieure oder Techniker hätten die entsprechenden Motive vermutlich ganz anders festgehalten. Die Künstler kannten sich mit der Materie oft nicht aus und waren überdies noch abhängig von ihren Auftraggebern und den Gesichtspunkten, unter denen diese ihre Unternehmen präsentieren wollten. ${ }^{8}$ Arbeiter hätten mit großer Wahrscheinlichkeit andere Motive ausgewählt.

Weiter gilt, dass Industriegemälde nicht nur im Zarenreich, sondern auch in den west- und nordeuropäischen Ländern gemessen an der absoluten Zahl der Gemälde eine Randerscheinung waren, die nicht zum klassischen Themenrepertoire zählten. Zwar gab es vereinzelt Künstler wie den französischen Maler François Bonhommé (1809-1891), der ab den 183oer Jahren regelmäßig Aufträge aus der Industrie, später auch vom französischen Staat, annahm, um Industriemotive abzubilden, doch dies waren Ausnahmen. ${ }^{9}$

Die Bilder von Arbeitsprozessen haben ihren Ursprung in der antiken Tradition der Monatsbilder und den mittelalterlichen Stundenbüchern, die berufsspezifische Arbeitsgänge und die im jeweiligen Monat typischen Tätigkeiten wiedergaben. Allerdings beschränkten sich diese Bilder vornehmlich auf das Leben der Handwerker und Bauern. Erst ab dem 17. und frühen 18. Jahrhundert wählten besonders Künstler aus Großbritannien und Schweden frühe Entstehungsformen von Industrie als Motive aus. ${ }^{10}$ Die Maler, wie beispielsweise der Engländer Joseph Wright of Derby (1734-1797), zeigten sich besonders fasziniert von Bergwerken und dem Spektakel des Feuers, von Rauch und Glut in der Eisenindustrie. ${ }^{11}$ Beliebt waren daher Szenen aus Schmiedewerkstätten, wobei diese noch stark an klassische Handwerksbetriebe erinnern. Insgesamt lässt sich beobachten, dass die Künstler erst mit einer gewissen Verzögerung auf

des 19. Jahrhunderts, in: Industriebilder aus Westfalen. Gemälde, Aquarelle, Handzeichnungen, Druckgrafik, 1800-1960. Westfälisches Landesmuseum für Kunst und Kulturgeschichte Münster Landschaftsverband Westfalen-Lippe, 7.9. bis 21.10.1979, Münster 1979, S. 11-18, S. 11.

7 Brandt: Schaffende Arbeit und Bildende Kunst, S. 331; Türk: Bilder der Arbeit, S. 18.

8 Klaus Türk: Vorbemerkungen des Herausgebers, in: ders. (Hrsg.): Arbeit und Industrie in der bildenden Kunst. Beiträge eines interdisziplinären Symposiums, Stuttgart 1997, S. 7-12, S. 10.

9 Türk: Bilder der Arbeit, S. 164-165.

$10 \quad$ Klingender: Kunst und industrielle Revolution, S. 57, 61.

11 Hoffmeister: Europäische Industriegemälde, S. 27-28. 
den Einsatz von Maschinen im industriellen Herstellungsprozess reagierten. Erst im 19. Jahrhundert entwickelten sie außerdem den Anspruch, sich in ihren Gemälden mit dem Alltag der Menschen auseinanderzusetzen und sich allen Lebensbereichen zu widmen. ${ }^{12}$ In der russischen Malerei traten Maschinen bis zum Ersten Weltkrieg kaum als Motiv in Erscheinung. Einer der Gründe hierfür dürfte gewesen sein, dass die Industrie im Zarenreich noch bis weit ins 19. Jahrhundert hinein wirtschaftlich eine eher untergeordnete Rolle spielte. ${ }^{13}$

Weitaus häufiger waren in der westeuropäischen Malerei Landschaftsansichten, in denen die Natur als Kulisse für das beeindruckende Schauspiel der Eisenverhüttung fungiert. Die Künstler wählten oft nächtliche Szenen, in denen dunkle Baumsilhouetten vor einem gelb-orangenen Feuerschein die Kraft der Elemente betonten, die sich der Mensch Untertan machte. ${ }^{14}$ Neben diesen Farbenspektakeln nutzten die Maler die Fabriken als pittoreske Elemente, die sie auf romantischen Landschaftsgemälden in die Szenerie integrierten..$^{15}$ Gerade die englischen Werke waren in Europa sehr einflussreich und inspirierten beispielsweise die französischen Darstellungen von Eisenhütten der $1830 e r$ bis $1850 e r$ Jahre. ${ }^{16}$ Sowohl in der Landschaftsmalerei als auch bei szenischen Abbildungen der Manufaktur- und Fabrikarbeit überwog jedoch bis zu Beginn des 19. Jahrhunderts die Faszination für die neue Technik. Den Künstlern reichte es, ihrem Publikum einen groben Eindruck von Maschinen und der Produktion zu geben, sie verfolgten nicht das Ziel, die Arbeitsschritte oder mechanischen Anlagen realistisch wiederzugeben. ${ }^{17}$

In der russischen Malerei spielte die Landschaftsmalerei bis in die $1820 \mathrm{er}$ Jahre kaum eine Rolle, weil russische Landschaften nicht als erhaben oder pittoresk galten. Dieses Charakteristikum war der westeuropäischen Natur vorbehalten, weswegen Maler wie Literaten bis ins 19. Jahrhundert russische Landschaften europäisierten, so dass die Ansichten teilweise stark an Italienbilder erinnerten. ${ }^{18}$ Erst als die Romantik das Zarenreich erreichte, etablierten

12 Marc Le Bot: Peinture et machinisme, Paris 1973, S. $75^{-77}, 83$.

13 Siehe: Kapitel „Fotografien und Industrie - Historischer Überblick“, S. 27-6o.

14 Klingender: Kunst und industrielle Revolution, S. 19. Zur Ästhetik archaischer Naturgewalt vor einem nächtlichen Hintergrund siehe auch: Georg Maag: Kunst und Industrie im Zeitalter der ersten Weltausstellungen. Synchrone Analyse einer Epochenschwelle, Konstanz 1982, S. 149-151.

15 Siegfried Salzmann: Einleitung, in: Wilhelm-Lehmbruck-Museum (Hrsg.): Industrie und Technik in der deutschen Malerei von der Romantik bis zur Gegenwart, Duisburg 1969, S. 9-11, S. 9 .

16 Pierrot: Les images de l'industrie en France, S. 346.

17 Türk: Bilder der Arbeit, S. 18.

18 In der Literatur fand die Umdeutung der russischen Landschaft als beschreibungswürdiges Motiv in den 1840er Jahren etwas früher statt als in der Malerei. Klaus Gestwa: 
sich Landschaftsbeschreibungen zunächst in der russischen Literatur, und bald wandten sich auch Maler diesem Genre zu. ${ }^{19}$ Zunächst orientierten sich die Künstler an Werken ihrer deutschen, französischen und niederländischen Kollegen. ${ }^{20}$ Dabei dominierten Darstellungen ausgefallener Naturphänomene wie Gewitter oder Stürme, mit denen die Maler ihrem Publikum bestimmte Emotionen zu vermittelten suchten. ${ }^{21} \mathrm{Zu}$ diesem Zweck hätten zwar auch Darstellungen von Industrieanalgen dienen können, doch fehlte es den russischen Malern in der ersten Jahrhunderthälfte an Anschauungsmaterial. Die ersten industriellen Produktionsanlagen im Zarenreich waren vorwiegend Manufakturen, die Textil- oder sonstige Konsumprodukte herstellten. ${ }^{22}$ Hier waren keine vergleichbar beeindruckenden Schauspiele wie bei der Eisenverhüttung zu beobachten. Zwar gab es Schmieden, diese waren jedoch alltägliche Erscheinungen und genügten nicht dem Anspruch der Romantiker, einen besonderen Moment festzuhalten. ${ }^{23}$ Erste Genreszenen, die besonders weibliche Arbeit darstellen, malte in Russland Vasilij Andreevič Tropinin (1776-1857). Auf seinen Gemälden „Die Spinnerin“ und „Die Spitzenklöpplerin“ bildete er im russischen Biedermeierstil ${ }^{24}$ jeweils eine arbeitende Frau und ihre Tätigkeit

Der Blick auf Land und Leute. Eine historische Topographie russischer Landschaften im Zeitalter von Absolutismus, Aufklärung und Romantik, in: Historische Zeitschrift, Jg. 279/2004, Heft 1, S. 63-125, S. 96-97, 109, 111.

19 Vladimir Lenyashin: Everything Gravitates towards the Landscape. Landscape Painting in the Context of the Russian Genre System, in: David Jackson; Patty Wageman (Hrsg.): Russian Landscape, Schoten 2003, S. 137-145, S. 137.

20 Henk van Os: Russian Landscapes. A Première, in: David Jackson; Patty Wageman (Hrsg.): Russian Landscape, Schoten 2003, S. 13-42, S. 10-21, 28.

21 Ljudmila Markina: „Kinder des Jahres 1812“. Die russische Malerei der Romantik und des Biedermeier, in: Kunst- und Ausstellungshalle der Bundesrepublik Deutschland (Hrsg.): Russlands Seele. Ikonen, Gemälde, Zeichnungen aus der staatlichen Tretjakow-Galerie, Moskau, Bonn 2006, S. 108-127, S. 108.

22 Winks; Neuberger: Europe and the Making of Modernity, S. 91. Auch in der französischen Malerei lässt sich beobachten, dass die Textilindustrie Maler nur selten zu Bildern inspirierte. Nicolas Pierrot: „À l'époque où l'ouvrier sévissait dans l'art ....". La représentation du travail industriel en France dans la peinture de chevalet, 1870-1914, in: o. A. (Hrsg.): Des Plaines à l'usine. Images du travail dans la peinture française de 1870 à 1914, Paris 2001, S. $96-113$, S. 107.

23 Markina: „Kinder des Jahres 1812“, S. 108.

24 In der sowjetischen Kunstgeschichte war es lange umstritten, ob es den Biedermeier als Stilrichtung überhaupt gegeben habe. Als erste gebrauchte N. N. Kovalenskaja in ihrer Dissertation von 1929 den Begriff. Dieser wurde jedoch erst wieder in den 1980er Jahren aufgegriffen. In der Zwischenzeit geht man in der Forschung davon aus, dass es den Stil gegeben habe. Ende der 1990er Jahre entstand eine Reihe von Dissertationen und Publikationen, die sich dem russischen Biedermeier zuwandten: Ljudmila A. Markina: Russische Malerei der Biedermeierzeit, in: Birgit Biedermann; Marianne Heinz; Hans 
ab. Allerdings handelte es sich auch hier um Darstellungen handwerklicher Arbeit - die Industrie spielte auch im russischen Biedermeier keine Rolle. ${ }^{25}$

Ein weiteres Motiv neben Arbeitsszenen und Landschaftsdarstellungen waren Besuche wichtiger Persönlichkeiten. $\mathrm{Zu}$ diesen Anlässen erhielten Maler den Auftrag, Gäste und Belegschaft auf dem Fabrikareal oder in einer Werkstatt malerisch festzuhalten. Die ersten Beispiele solcher Bilder stammen aus Frankreich und entstanden Ende des 18. Jahrhunderts. Allerdings zeigen diese frühen Darstellungen fast ausschließlich Besucherdelegationen und Arbeiter unter freiem Himmel vor den Fabrikgebäuden. Dies hing vermutlich damit zusammen, dass die Künstler keinen Zugang ins Innere der Betriebe erhielten. Im Zarenreich waren Gemälde von Herrscherbesuchen nicht üblich, obwohl die Zaren immer wieder gerade staatliche Betriebe besichtigten. Offenbar entsprachen diese Motive nicht den Ansprüchen der monarchischen Selbstinszenierung. ${ }^{26}$

Insgesamt dominierte in der Frühzeit der Industrialisierung eine positive Haltung gegenüber der neuen Entwicklung. Diese Faszination wandelte sich besonders in Großbritannien bereits um die Wende zum 19. Jahrhundert. Zunehmend meldeten sich kritische Stimmen in den Diskussionen zu Wort und prangerten Missstände wie Kinderarbeit an. ${ }^{27}$ Gerade englische und französische Literaten blickten oft skeptisch oder ablehnend auf die Industrialisierung und ihre sozialen Auswirkungen. ${ }^{28}$ Auch unter den Malern gab es Kritiker der neuen Entwicklung wie den englischen Künstler John Martin (1789-1854). Laut Francis Donald Klingender verarbeitete Martin seine Erfahrungen aus der Arbeit in englischen Mienen, indem er Höllen- und Weltuntergansszenarien malte. ${ }^{29}$ Dennoch blieben in der ersten Hälfte des 19. Jahrhunderts kritische Abbildungen der Industrie innerhalb der Malerei eher selten.

In der zweiten Hälfe des 19. Jahrhunderts wandelte sich die Haltung der Maler gegenüber dem Wachstum der Industrie und den damit einhergehenden gesellschaftlichen Veränderungen. Dabei war die Situation in verschiedenen

Ottomeyer (Hrsg.): Russische Malerei der Biedermeierzeit. Meisterwerke aus der Tretjakow-Galerie Moskau im Dialog mit Gemälden der Neuen Galerie Kassel, Eurasburg 1999, S. 13-19, S. 15 .

25 Markina: „Kinder des Jahres 1812“, S. 126.

26 Weitere Details siehe: Kapitel „Die Fabrik wird salonfähig - der Erste Weltkrieg“, S. 325-368.

27 Zu Diskussionen über Kinderarbeit im Zarenreich siehe: Boris Gorshkov: Russia's Factory Children. State, Society, and Law, 1800-1917, Pittsburgh 2009.

28 Pierrot: Les images de l'industrie en France, S. 23.

29 Klingender: Kunst und industrielle Revolution, S. 109-111. 
europäischen Ländern sehr unterschiedlich. Besonders sozialkritisch war die Malerei in Belgien. Die Künstler widmeten sich hier insbesondere der Montanindustrie und zeigten aus Sicht der betroffenen Arbeiterinnen und Arbeiter deren harte Arbeitsbedingungen. ${ }^{30}$ Dabei betonten die Kunstschaffenden die Würde der Menschen, die sie als Personen aus einer anderen gesellschaftlichen Schicht darstellten, die dem Betrachter dennoch ebenbürtig gegenüberstanden. Deutsche Maler vertraten eine weniger eindeutige Haltung gegenüber der Industrialisierung. Als Beispiel kann Menzels „Eisenwalzwerk“ gelten, das sowohl als heroische Industriedarstellung als auch nach einer sozialkritischen Lesart interpretiert wurde. Es entstanden jedoch auch Gemälde, die die Industrie als Segen für die Menschheit feierten. ${ }^{31}$

In Frankreich widmeten sich besonders die Impressionisten ab den 186oer Jahren den neuen Industrielandschaften. Auf der ersten Gruppenausstellung der Impressionisten 1874 zeigten beispielsweise drei von fünf Werken Claude Monets (1840-1926) wirtschaftliche Aktivität und Industrielandschaften. ${ }^{32}$ In der französischen Malerei hatte es um die Mitte des Jahrhunderts einen Trend zum Realismus gegeben, in dem Landschaften als nostalgische Rückzugsorte galten. Im Gegensatz dazu lag den Impressionisten daran, die Szenerie so wiederzugeben, wie sie sich ihnen präsentierte. ${ }^{33}$ Folglich waren Zeichen industrieller Entwicklungen wie Eisenbahnlinien, Brücken und noch häufiger Fabriken und Schornsteine Teil der Bildkompositionen. ${ }^{34}$ Dabei waren die rauchenden Schornsteine neben einem ästhetischen Element auch ein Symbol für industrielle Produktivität. ${ }^{35}$ In den Niederlanden wiederum standen um 1870 die Mitglieder der Haager Schule den technischen Neuerungen der Industrialisierung zwiespältig gegenüber. Die Künstler griffen in ihren realistischen Gemälden nur selten die gesellschaftlichen Umwälzungen auf, sondern widmeten sich vorwiegend der Darstellung der Natur. ${ }^{36}$

$30 \quad$ Wichtige Vertreter dieser Richtung waren zum Beispiel Léon Frédéric (1856-1940); Cécile Douard, eigentlich Cécile Leseine (1866-1941) und Constantin Meunier (1831-1905).

31 Türk: Bilder der Arbeit, S. 172-175, 180-182.

32 James Rubin: Impressionism and the Modern Landscape. Productivity, Technology, and Urbanization from Manet to van Gogh, Berkeley, Los Angeles, London 2008, S. 1.

33 Die Impressionisten legten besonderen Wert auf Atmosphäre und Licht. Diese fingen sie mit ihrer charakteristischen Technik von punkt- oder kommaartig aneinandergereihten Pinselstrichen ein. Es ging ihnen nicht darum, besonders schöne Landschaften darzustellen. Daher verzichteten sie darauf, Schornsteine und Industrieanlagen auszusparen.

34 Rubin: Impressionism and the Modern Landscape, S. 121-125.

35 Rubin: Impressionism and the Modern Landscape, S. 130.

36 Jenny Reynaerts: Die Ästhetik der Polderlandschaft, in: dies. (Hrsg.): Der weite Blick. Landschaften der Haager Schule aus dem Rijksmuseum, München 2008, S. 39-67, S. 39, 47-49. 
Im Zarenreich war es besonders eine Gruppe junger Künstler, später bekannt als peredvižniki (Die Wanderer), die in den 186oer und 1870er Jahren eine neue Entwicklung in der russischen Malerei initiierte. Ihre Mitglieder waren geprägt durch die Folgen der revolutionären Bewegungen der 1840er Jahre, den verlorenen Krimkrieg (1853-1856) sowie die Bauernbefreiung (1861) und die Großen Reformen, die Alexander II. veranlasst hatte. Viele dieser Künstlergeneration stammten aus einfachen Verhältnissen und fühlten sich der einfachen Bevölkerung darum besonders verbunden. Sie griffen einerseits in Genrebildern das Leben derjenigen auf, die von der russischen Malerei bislang vergessen worden waren, wie einfache Beamte, Kaufleute oder Bauern. ${ }^{37}$ Die neuen Produktionsmethoden in Fabriken waren jedoch auch bei ihnen kein beliebtes Motiv. Auch der "Heizer" (1878) von Nikolaj Aleksandovič Jaroščenko (1846-1898) lässt offen, ob es sich um einen revolutionären Fabrikarbeiter oder um einen traditionellen Handwerker in einer Schmiede handelt. ${ }^{38}$ In den realistischen Bildern prangerten die Maler soziale Missstände an. Sie hatten die Hoffnung, die Menschen mit ihren Gemälden dazu zu bewegen, aktiv zu werden und ihre Lebensverhältnisse zu verändern. ${ }^{39}$ Damit möglichst viele Menschen ihre Bilder sahen, schlossen sich die Künstler 1870 zur Gesellschaft für Wanderausstellungen (Tovariščestvo peredvižnych chudožestvennych vystavok) zusammen und organisierten im folgenden Jahr die erste Ausstellung, die in St. Petersburg und Moskau sowie in Kiew, Charkow und Odessa zu sehen war. ${ }^{40}$ Das imaginierte Publikum, für das die Maler ihre Werke entwarfen, waren die Bauern, die im Verständnis der Künstler das russische Volk repräsentierten. Neben Genrebildern entwickelten Mitglieder der Künstlergruppe auch eine neue Form der Landschaftsmalerei, bei der die Personen aus den Gemälden verschwanden. Stattdessen blickt der Betrachter aus der Perspektive der Landbevölkerung auf die Szenerie. ${ }^{41}$

37 Galina Tschurak: Auf der Suche nach Russland. Die Wanderer und ihre Themen, in: Kunst- und Ausstellungshalle der Bundesrepublik Deutschland (Hrsg.): Russlands Seele. Ikonen, Gemälde, Zeichnungen aus der staatlichen Tretjakow-Galerie, Moskau, Bonn 2006, S. 140-171, S. 142.

38 Christoph Schmidt: Vom Messias zum Prolet. Arbeiter in der Kunst, Stuttgart 2010, S. $38-39$.

39 Tschurak: Auf der Suche nach Russland, S. 146.

40 Ernst-Gerhard Güse: Zwischen St. Petersburg, Rom, Paris und Moskau. Russische Landschaftsmalerei nach 1860, in: Sabine Fehlemann (Hrsg.): Ilja Repin und seine Malerfreunde. Russland vor der Revolution, Wuppertal 2005, S. 23-30, S. 23; Tschurak: Auf der Suche nach Russland, S. 140.

41 Christopher Ely: This Meager Nature. Landscape and National Identity in Imperial Russia, DeKalb 2002, S. 217-218. 
Sowohl das neu erwachte Interesse am einfachen Volk als auch die Begeisterung für die Natur waren kein spezifisches Merkmal der russischen Malerei. In ganz Westeuropa gewannen in der zweiten Hälfte des 19. Jahrhunderts nationale Ideen und Identitäten an Bedeutung. ${ }^{42}$ In diesem $\mathrm{Zu}$ sammenhang spielte die Natur eine wichtige Rolle, denn mit der Frage nach einer nationalen Identität entstand das Bedürfnis, auch eine nationale Landschaft $\mathrm{zu}$ erschaffen. ${ }^{43}$ An diesen Diskussionen beteiligten sich in erster Linie Menschen, die bereits in Städten lebten, so dass im Zuge dessen eine Exotisierung der Natur einsetzte. ${ }^{44}$ Es waren besonders die Gedanken Jean-Jacques Rousseaus (1712-1778), die die Suche nach einer nationalen Identität im Zarenreich beeinflussten. ${ }^{45}$ Der Philosoph hatte eine Hinwendung zur Natur und dem Natürlichen propagiert. Industrie und die Lebensbedingungen von Arbeitern spielten für ihn keine Rolle. Auch bei der Suche nach einer russischen Identität hatten sie keine Bedeutung. Fabriken waren künstlich hergestellte, aus Westeuropa importierte Orte. Sie waren das Gegenteil der nordrussischen Landschaften. Inbegriff und Höhepunkt der Landschaftsmalerei waren die von Ivan Ivanovič Šiškin (1832-1898) oder Isaak Il'ič Levitan (1860-1900) geschaffenen Gemälde. ${ }^{46}$ Zwar gab es in den 187oer Jahren mehr und mehr Industrieanlagen, und die Arbeiter versuchten in ersten Streiks, ihre Interessen gegenüber den Unternehmern durchzusetzen, ${ }^{47}$ doch die Künstler sahen den eigentlichen Schlüssel für die Identität und Zukunft Russlands in den Bauern und deren traditioneller Lebensweise. ${ }^{48}$ Diese Leitlinie erreichte ihren Höhepunkt in den $1870 e r$ Jahren, bestimmte die russische Malerei aber noch bis zur Jahrhundertwende. In den $1890 e r$ Jahren gewann eine neue Künstlergeneration an Einfluss. Ihre Mitglieder lehnten das sozialkritische Pathos der Wanderer ab und wollten stattdessen nur die schönen Seiten des Lebens darstellen. ${ }^{49}$ Es verwundert folglich nicht, dass auch auf ihren Gemälden

42 Hierzu beispielsweise: Dieter Langewiesche: Nation, Nationalismus, Nationalstaat in Deutschland und Europa, München 2000.

43 Ely: This Meager Nature, S. 5; Lenyashin: Everything Gravitates towards the Landscape, S. 140 .

44 Reynaerts: Die Ästhetik der Polderlandschaft, S. 40-50.

45 Dmitrii S. Likhachev: Reflections on Russia, Boulder Colo. u. a. 1991, S. 16.

46 Gertrud Pickhan: „Lewithanisierende Rußlandsucher“. Isaak Levithan (1860-190o) und die zeitgenössische Rezeption seines Werks, in: Jahrbücher für Geschichte Osteuropas, Jg. 6o/2012, Heft 4, S. 591-616, S. 611, 614.

47 Hierzu beispielsweise: Reginald E. Zelnik: Law and Disorder on the Narva River. The Kreenholm Strike of 1872, Berkeley 1995.

48 Orlando Figes: Nataschas Tanz. Eine Kulturgeschichte Russlands, Berlin 2011, S. 257.

49 Tschurak: Auf der Suche nach Russland, S. 170. 
Arbeiter und Fabriken keinen Platz fanden, obwohl es jetzt mehr Anschauungsmaterial gab als je zuvor.

\section{2 $\quad$ Abseits der großen Formate}

Die meisten Autoren von Untersuchungen zur Industriemalerei betonen, dass es im 18. und 19. Jahrhundert nur wenige Bilder von Industrieanlagen gegeben habe. Dem widerspricht Nicolas Pierrot, der in seiner Dissertation Fabrikdarstellungen in Frankreich von 1760 bis 1870 untersucht. Allein für diesen Zeitraum konnte er mehrere tausend Abbildungen ausfindig machen, wobei er Zeichnungen, Presseillustrationen oder Plakate nicht einbezog. ${ }^{50} \mathrm{Im}$ Unterschied zu anderen Forschungsarbeiten beschränkt sich Pierrot nicht auf Gemälde, sondern untersucht auch Stiche und Lithografien. Diese Drucke waren verglichen mit Ölgemälden billiger ${ }^{51}$ und entsprechend weniger wertvoll. Infolgedessen gingen die Darstellungen schneller verloren, und es existieren in Frankreich heute kaum geschlossene Bildkorpora dieser Abbildungen, was die wissenschaftliche Arbeit erschwert. ${ }^{2}$ Die Situation in der Russischen Föderation stellt sich ähnlich dar. Zwar existieren Druckgrafiken aus dem Zarenreich, die Fabriken zeigen, diese sind jedoch meist weit zerstreut. ${ }^{53}$ Auch widmete sich bislang keine Untersuchung systematisch diesen Abbildungen. Im Folgenden werden darum ein erstes Mal die Forschungsergebnisse Pierrots mit der Sammlung aus der Rossijskaja Gosudarstvennaja Biblioteka (Russische Staatsbibliothek) verglichen und herausgearbeitet, in welchen Punkten sich die Entwicklung von Fabrikdarstellungen in beiden Ländern deckte und welche Unterschiede es gab.

5o Nicolas Pierrot: Le silence des artistes? Thématique industrielle et diversification des supports (v. 1850-fin XIXe siècle), in: Denis Woronoff (Hrsg.): Les images de l'industrie de 1850 à nos jours. Actes du colloque tenu à Bercy, le 28 et 29 juin 2001, Paris 2002, S. 10-20, S. 13 .

$5^{1}$ In Frankreich kostete 1823 ein Stich ca. einen Franc. Dies war zwischen 1832-1835 der durchschnittliche Tageslohn eines Webers am Hochrhein und etwas weniger als der Lohn eines Stoffdruckers aus derselben Region. Pierrot: Les images de l'industrie en France, S. 215.

52 Pierrot: Les images de l'industrie en France, S. 8, 54-55.

53 Im Rahmen dieser Arbeit war es nicht möglich, eine systematische Recherche durchzuführen. Sicherlich lassen sich jedoch in Bibliotheken sowie in den Sammlungen der großen Kunstmuseen, aber auch in Heimat- und Firmenmuseen entsprechende Druckgrafiken finden, welche eine aussagekräftige Quellenbasis für eine vielversprechende Analyse bilden könnten. 
In Frankreich entstanden die ersten Industriedarstellungen Ende des 17. Jahrhunderts, sie waren jedoch noch sehr selten. Von 1755 bis 1789 waren Fabriken und Manufakturen nur dann abbildungswürdig, wenn sie den Ruhm des Empire dokumentierten. Erst ab der Wende zum 19. Jahrhundert erstellten Künstler öfter Ansichten von Betrieben, ab 1822 waren diese regelmäßig im Salon ausgestellt.. ${ }^{54}$ Im Zarenreich kamen Drucke von Fabrikgebäuden nach bisherigem Wissensstand erst im 19. Jahrhundert auf. Vermutlich hatte es bis dahin zu wenige Beispiele entsprechender Einrichtungen gegeben, als dass sie Grafiker in großem Stil inspiriert hätten. Die Sammlung der Staatsbibliothek umfasst zwölf Stiche und Lithografien, von denen jedoch nur zwei eindeutig datiert sind, auf 1855 und $1864 / 67$. Zwei weitere Stiche entstanden vermutlich $1845 .{ }^{55}$ Pierrot stellt Perioden fest, in denen Industriedarstellungen besonders beliebt waren. Er gibt jedoch zu bedenken, dass diese Trends auch damit zusammenhängen, wann die großen Druckgrafik-Sammlungen französischer Landschaften zusammengestellt wurden. ${ }^{56}$ Es handelte sich hierbei um eine europäische Mode, beispielsweise entstanden auch in Belgien um die Jahrhundertmitte entsprechende Serien. ${ }^{57}$ Für das Zarenreich sind jedoch keine vergleichbaren Sammlungen bekannt. Dennoch gleichen sich einige Drucke der untersuchten Sammlung auffallend, so dass davon ausgegangen werden kann, dass sie als Serie erschienen und möglicherweise von dem selben Künstler stammen. ${ }^{58}$

Die meisten Motive zeigen Außenansichten der Fabriken inmitten der sie umgebenden Landschaft (Abb. 1).

Pierrot sieht diese Darstellungen in der Tradition der Vedutenmalerei, bei der die Maler den Reichtum einer bestimmten Region untermauern sollten. Der Begriff „Vedute“ bedeutet das Gesehene, das, was man sieht. ${ }^{59}$ Damit beschreibt der Name das Merkmal der Darstellungen, denn bei Veduten handelt es sich um an topographische Gegebenheiten angelehnte Landschaftsdarstellungen. Die Maler ließen sich nicht von imaginären Szenarien inspirieren, sondern fertigten Abbildungen realer Ansichten mit jeweils regionalen Spezifika an,

54 Pierrot: Les images de l'industrie en France, S. 6, 72, 76.

55 http://library.krasno.ru/Pages/Kraevedenie/Voznesenka19.htm (zuletzt eingesehen am 29.09.15); http://bagira.guru/rodina/1992-04/stroptivyy-kankrin.html?fontstyle=f-larger (zuletzt eingesehen am 29.09.15).

56 Pierrot: Les images de l'industrie en France, S. 100.

57 Bart Van der Herten; Michel Oris; Jan Roegiers (Hrsg.): La Belgique industrielle en 1850. Deux cents images d'un monde nouveau, Bruxelles 1995 .

58 RGB IZO: $63175-44 ; 63176-44 ; 63178-44 ; 63180-44$, sowie die Drucke RGB IZO: 11234649; 112347-49; 112348-49; 112349-49.

59 o. A.: Art. „Vedute“, in: Wolf Stadler (Hrsg.): Lexikon der Kunst. Malerei, Architektur, Bildhauerkunst, Bd. 12, Freiburg 1990, S. 107-111, S. 107. 


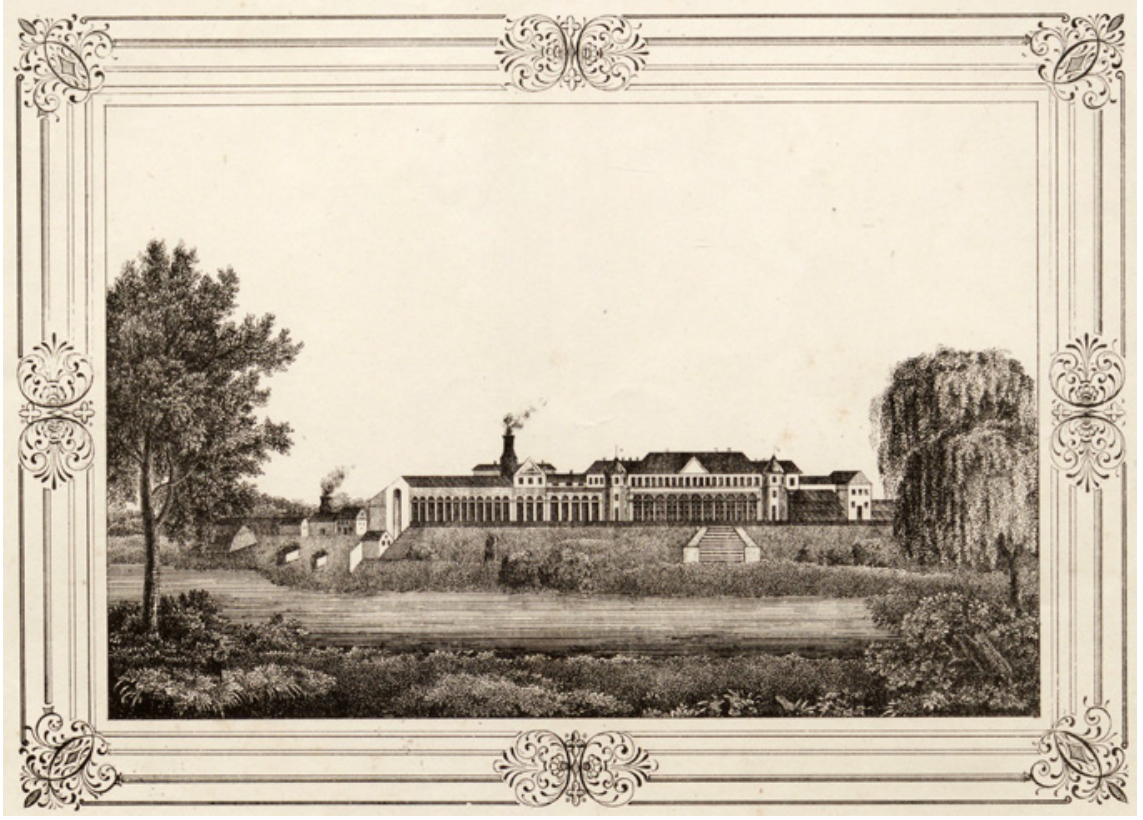

Abb. 1

N. A. Volkov: Gorinskaja Papierfabrik (Gorinskaja Bumagoprjadil'naja fabrika), o. O. o. J. RGB $63180-44$

wobei sie zur besseren Wirkung ihre Bilder bewusst arrangierten. ${ }^{60}$ Dabei galten Natur, Kulturlandschaft, Gebäudestrukturen sowie Ortsbilder als unzertrennliche Einheit. ${ }^{61}$ Trotz der im 19. Jahrhundert beliebten romantischen Ausgestaltung des Genres mussten die Betrachter immer erkennen können, um welche Ansicht es sich handelte. Dies machte Veduten mit der zunehmenden Mobilität und Reisetätigkeit der Menschen im 19. Jahrhundert zum idealen Souvenir. ${ }^{62}$

Speziell für die Gesamtansichten von Fabriken sieht Pierrot weitere Vorläufer in den Schlossansichten des 18. Jahrhunderts, ${ }^{63}$ auch Schlossveduten

6o Hermann Voss: Studien zur venezianischen Vedutenmalerei des 18. Jahrhunderts, in: Repertorium für Kunstwissenschaft 47, Leipzig 1926, S. 1-45, S. 1.

61 Benno Lehmann: Die Stadt- und Landschaftsvedute im 19. Jahrhundert, in: Eberhard Knittel (Hrsg.): Veduten des 19. Jahrhunderts Vignetten zum Goethe-Jahr (= In Baden-Württemberg: Kultur, Leben, Natur, Jg. 29/1982, Heft 1), S. 7-9, S. 7.

62 Lehmann: Die Stadt- und Landschaftsvedute im 19. Jahrhundert, S. 8. Diese Funktion übernahmen in der zweiten Hälfte des 19. Jahrhunderts vermehrt Fotografien und Postkarten. o. A.: Art. „Vedutenmalerei“, in: Harald Olbrich u. a. (Hrsg.): Lexikon der Kunst. Architektur, bildende Kunst, angewandte Kunst, Industrieformgestaltung, Kunsttheorie, Bd. 7, Leipzig 1994, S. 570-571.

63 Pierrot: Les images de l'industrie en France, S. 125. 
genannt. ${ }^{64}$ Diese Traditionslinien prägen auch die Abbildung der russischen Gorinskaja Papierfabrik (Abb. 1). Auf den niederländischen und italienischen Vorbildern der Veduten nutzten die Künstler oft Bäume, um ihre Landschaften einzurahmen. Diese Funktion übernehmen im vorliegenden Stich eine Trauerweide und ein nicht näher bestimmbarer Baum, die groß im Vordergrund stehen. Beide unterstreichen den relativ symmetrischen, klassischen Bildaufbau. Die repräsentative Treppe führt den Blick des Betrachters zum Hauptgebäude des Unternehmens, das mit seinen Arkaden und Türmchen selbst stark an ein Schloss erinnert. Vergleichbar mit den französischen Grafiken lässt das Gebäude kaum Rückschlüsse darauf zu, dass es zur Papierherstellung diente. Allein die beiden Rauchwölkchen deuten dies an. Rauch hatte sich auf Stichen schon früh zum Symbol für Aktivität und Produktivität entwickelt. ${ }^{65}$

Im Unterschied $\mathrm{zu}$ den Bäumen und Pflanzenelementen muten die pittoresken Rauchkringel auf Abbildung 1 allerdings wenig natürlich an. Auch die Gebäude wirken sehr schematisch, als habe der Künstler sie nicht während eines Ortsbesuchs gezeichnet, sondern am Reißbrett entworfen. Fast scheint es, als habe der Grafiker keine Erfahrung mit der Abbildung von Industrieanlagen gehabt. Dagegen wirkt die Lithographie der Textilfabrik der Brüder Gučkovyj (Abb. 2), die ca. zehn Jahre später entstand, deutlich realistischer.

Obwohl Paškin den Baumbestand weniger detailreich abbildete, wirkt die Darstellung ausgewogener und dynamischer, der Betrachter kann sich problemlos den kräftigen Wind vorstellen, den der Rauch sowie die Fahne auf dem Türmchen links im Bild andeuten. Um das ästhetische Gleichgewicht ober- und unterhalb des Horizonts sicherzustellen, ließ der Künstler den Himmel nicht weiß (wie in Abb. 1), sondern deutete die Struktur der Wolken an. ${ }^{66}$ Auch dadurch wirken die Fabrikgebäude weniger schematisch, obwohl es sich mehrheitlich um rechteckige Gebäudekomplexe handelt.

Allen Drucken der analysierten Sammlung ist gemein, dass sie eine neutrale bis positive Sicht auf die Industrie wiedergeben. Damit unterscheiden sie sich von französischen Abbildungen aus der gleichen Zeit. In Frankreich verschwanden bereits in den $1830 e r$ Jahren allmählich die romantischen Einflüsse aus den Landschaftsdarstellungen. Die Künstler wählten dunklere Farbtöne, um den Raum wiederzugeben, und teilweise zeigten sie sogar Bäume, die ihre Blätter verloren hatten. ${ }^{67}$ Eine mögliche Erklärung für diese Beobachtung ist,

64 Sigrid-Jutta Motz: Fabrikdarstellungen in der deutschen Malerei von 1800 bis 1850 , Frankfurt a. M. 1980, S. 34-35.

65 Pierrot: Les images de l'industrie en France, S. 131-132.

66 Pierrot: Les images de l'industrie en France, S. 187-188.

67 Pierrot: Les images de l'industrie en France, S. 191. 


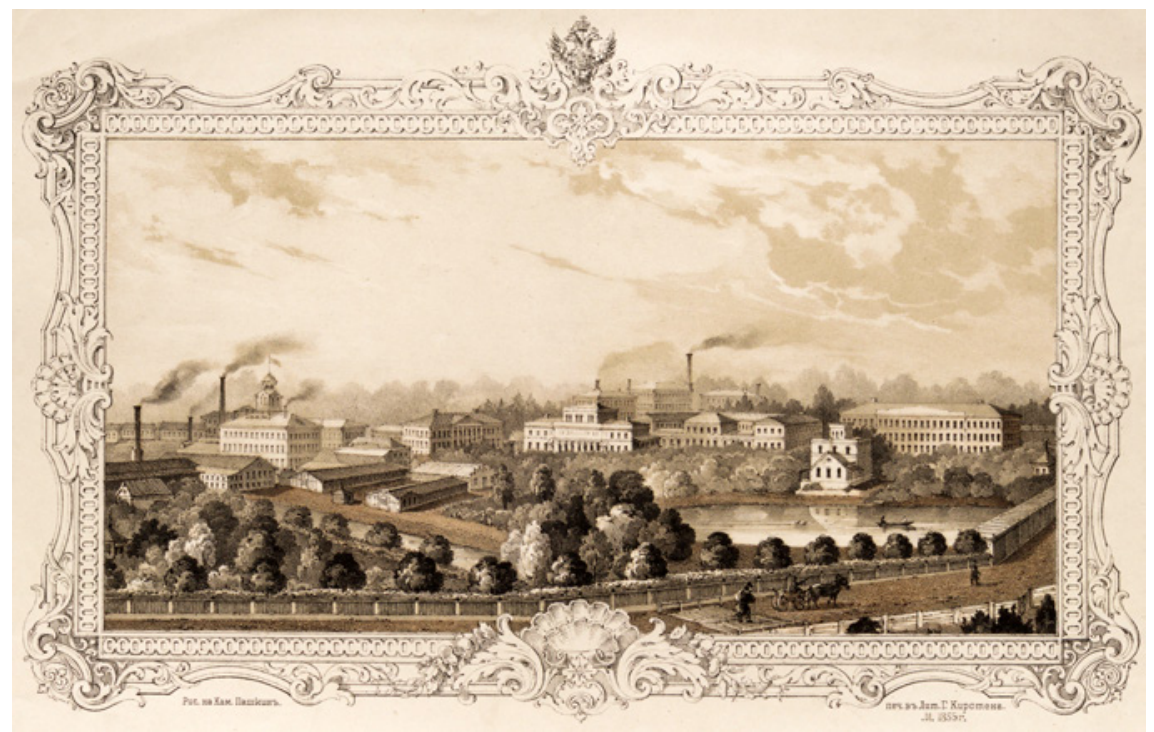

Abb. 2

Paškin: Fabrik der Brüder Gučkovyj (Fabrika Brat: Gučkovych), Lithographie (ris. na kam.), Moskva 1855. RGB 112351-49

dass es zu dieser Zeit im Zarenreich noch zu wenige Fabriken gab, damit die negativen Auswirkungen der Industrie für die Natur entsprechend gravierend ausgefallen wären. Denkbar ist auch, dass die russischen Künstler sich sehr stark an den romantischen Vorbildern ihrer deutschen, französischen oder niederländischen Kollegen orientierten. ${ }^{68}$ Bis in die $1820 e r$ Jahre hatte die russische Landschaftsmalerei nicht existiert und noch bis Mitte des Jahrhunderts war sie nur in Ansätzen vorhanden. Dieser geringe Rückhalt innerhalb der Malerei und die Suche nach einer eigenen Ausdrucksform könnten erklären, warum russische Kunstschaffende so stark an romantischen Darstellungen hingen und erst langsam auf kritische visuelle Darstellungen reagierten.

In anderen Bereichen eigneten sich die russischen Künstler dagegen relativ schnell neue Darstellungsweisen an. So integrierten französische Grafiker um $185^{\circ}$ nur dann Figuren, wenn diese die Szenerie belebten und mit Anekdoten bereicherten. ${ }^{69}$ Abbildung 2 zeigt beispielhaft, wie dieser Kunstgriff im Zarenreich zum Einsatz kam. Paškin zeigt auf dem Weg im Vordergrund ein Fuhrwerk und zwei Einzelpersonen, darüber hinaus sind auf dem Weiher ein Ruderboot mit zwei Passagieren und dahinter zwei Enten zu erkennen. Eine zweite Entwicklung betraf die Perspektive: Ende der 1840er Jahre

68 van Os: Russian Landscapes, S. 10-21, 28.

69 Pierrot: Les images de l'industrie en France, S. 83. 
zeigten französische Drucke vermehrt Fabriken aus der Vogelperspektive. ${ }^{70}$ Auch auf Paškins Ansicht der Textilfabrik blickt der Betrachter von einem erhöhten Punkt auf die Anlage. Dadurch sind hintereinander liegende Gebäude besser zu erkennen, und es ist für das Publikum einfacher, einen Eindruck des gesamten Firmengeländes zu bekommen. L(undwig) Pietzsch radikalisiert auf seiner Darstellung der Textilfabrik Gjubner diese Perspektive weiter, indem er einen Standpunkt schräg über der Anlage wählt, von dem aus der Zuschauer auf das Firmengelände blickt. In diesen beiden Fällen handelte es sich weniger um genuin neue Motive, sondern um die Erweiterung der bereits vorhandenen Darstellungsweise. Diese Innovationen übernahmen die Grafiker und Zeichner ohne große Vorbehalte von ihren europäischen Kollegen.

Pierrot zeigt für Frankreich, dass die Forschungsmeinung, es habe keine Darstellung von Fabriken und Industrieanlagen gegeben, nicht stimmt. Die Fallstudie anhand der Bestände der Russischen Staatsbibliothek hat gezeigt, dass Fabriken und Industrieanlagen auch in den Bildwelten des Zarenreichs ihren Platz hatten, wenn auch lange nicht innerhalb der Kunstmalerei. In einer Periode, in der Fabriken auf Gemälden keinen Platz fanden, waren Lithografien und Stiche diejenigen Kunstformen, mit der russische Künstler die neuen Erscheinungen festhielten. Diese Darstellungsformen erlebten einen Aufschwung im Rahmen der Weltausstellungen, für die Fabrikanten ganze Bilderserien in Auftrag gaben. Schließlich führte jedoch der Siegeszug der Fotografie dazu, dass die Druckgrafiken auch im Zarenreich ihre Bedeutung für die Abbildung von Fabriken einbüßten. ${ }^{71}$

\subsection{Russische Fabriken als Teil der visuellen Kultur}

Konflikte und Arbeitskämpfe nahmen im Laufe des 19. Jahrhunderts in ganz Europa zu, bekamen aber erst kurz vor der Jahrhundertwende einen Platz im Bilderkanon. ${ }^{72}$ In Frankreich zeigten zwar während der Wirtschaftskrise von 1873 bis 1896 immer wieder einzelne Gemälde des Salons streikende Arbeiter, doch das Publikum feindete diese Darstellungen häufig an. ${ }^{73}$ Es waren in erster Linie Grafiken und Karikaturen, mit denen Künstler Kritik an den Produktionsverhältnissen äußerten. Diese Abbildungen erschienen jedoch häufig in politisch linken Periodika und wandten sich damit an andere Betrachter als

70 Pierrot: Les images de l'industrie en France, S. 236.

71 Pierrot: Les images de l'industrie en France, S. 9, 237.

72 Türk: Bilder der Arbeit, S. 21.

73 Pierrot: „À l'époque où l'ouvrier sévissait dans l'art ....", S. 103. 
die klassischen Ölgemälde. ${ }^{74}$ Dies galt ebenso für das Zarenreich, wo in der Karikaturenzeitschrift Budil'nik (Der Wecker) bereits um die Jahrhundertwende industriekritische Zeichnungen erschienen. Allerdings wandte sich dieser subversive, kritische Ton nicht gegen einen offiziellen Bilddiskurs in der Malerei, der die Industrie gefeiert hätte. Denn auch um die Jahrhundertwende waren Fabriken und Produktionsanlagen in der russischen Malerei noch so gut wie nicht existent. Eine Ausnahme waren die Bilder von Mstislav Valerianovič Dobužinski (1875-1957), der sich bereits Anfang des 20. Jahrhunderts mit dem Konflikt zwischen Menschen und moderner Stadt auseinandersetzte. ${ }^{75}$ Auf seinen von emotionaler Kälte geprägten Stadtansichten spielen Fabriken zwar keine zentrale Rolle, ihre Schornsteine verstärken jedoch die trostlose, urbane Atmosphäre.

Eine zweite Ausnahme bilden die Gemälde von Nikolai Alekseevič Kasatkin (1859-1930), der als ein Wegbereiter des Sozialistischen Realismus gilt. ${ }^{76}$ Der Künstler war Mitglied der Wanderer und verarbeitete in seinen Genrebildern bereits ab Mitte der 18goer Jahre Eindrücke aus dem Leben der Bergleute und Arbeiter aus dem Donezbecken. ${ }^{77}$ Es entstanden Darstellungen wie 1893 Dve rabotnicy (Zwei Arbeiterinnen) oder 1894 Šachterka (Bergarbeiterin), auf denen die Protagonistinnen fröhlich lächeln. Andere Bilder wirken eher wie Studien eines Berufsstandes beispielsweise aus dem Jahr 1894 Šachter-zarubščik (Bergarbeiter Schrämer), 1895 Šachter s lampočkoj v rukach (Bergmann mit einem Lämpchen in den Händen) oder Sidjaščij šachter (Sitzender Bergmann) ebenfalls aus den 189oer Jahren. Kasatkin schönte die Menschen nicht, sondern zeigte ihre vom Kohlestaub dunkle Kleidung und Haut sowie die Spuren der schweren Tätigkeit unter Tage. Das harte Leben der Fabrikarbeiter fing der Künstler um die Jahrhundertwende im Gemälde Žena zavodskogo rabotčego (Frau eines Fabrikarbeiters) ein. Mit einem Säugling im Arm sitzt die Frau etwas zusammengesunken in der Mitte des Bildes und blickt nach rechts auf einen Punkt außerhalb der Darstellung. Um sie herum ist ein schlammiger Platz zu sehen mit schemenhaft angedeuteten Brettern, Balken und Steinen,

74 Türk: Bilder der Arbeit, S. 210, 252.

75 John E. Bowlt: Moskau und St. Petersburg. Kunst, Leben und Kultur in Russland, 190o1920, Wien 2008, S. 102; Irina Schumanowa: Mstislaw Dobuschinski, in: Kunst- und Ausstellungshalle der Bundesrepublik Deutschland (Hrsg.): Russlands Seele. Ikonen, Gemälde, Zeichnungen aus der staatlichen Tretjakow-Galerie, Moskau, Bonn 2006, S. 348.

$7_{6}$ Angelina Lucento: The Conflicted Origins of Soviet Visual Media. Painting, Photography, and Communication in Russia, 1925-1932, in: Cahiers du monde russe. Russie, Empire Russe, Union Soviétique, Etats Indépendants, 56/2015, Heft 2-3, S. 401-428, S. 408-409.

77 Diese hatte er während mehrerer Reisen gesammelt. Peter Leek: Russian Painting, New York 2012, S. 161. 
möglicherweise altes Baumaterial. Fröhlichkeit oder Zuversicht fehlen hier völlig.

Kasatkin war einer der wenigen Maler, der vor 1917 auch hinter die Fabriktore blickten. So auf den beiden Gemälden aus dem Jahr 1904 Tkačicha (Weberin) und Novyj tkackij zal. Orechovo-Zuevo (Neuer Websal. Orechovo Zuevo), auf denen Textilarbeiterinnen vor mechanischen Webstühlen zu sehen sind.

Es waren schließlich die revolutionären Ereignisse des Jahres 1905 und mit großer Wahrscheinlichkeit die Aufhebung der Zensur, die halfen, die Fabrik über Einzelfälle hinaus als Motiv im offiziellen Bilderkanon der russischen Malerei zu etablieren. 1906 entstand eine Reihe von Werken, auf denen sich Künstler Streiks und Protesten zuwandten. Beispielsweise malte Boris Michajlovič Kustodiev (1878-1927) einen Demonstrationszug anlässlich des Ersten Mais. Die Arbeiter mit ihren roten Fahnen strömen aus den Gebäuden der Putilovwerke, die im Hintergrund zu erkennen sind. ${ }^{78}$ Während bei Kustodiev die Demonstration einen geordneten Eindruck macht, zeigen die Gemälde Ataka zavoda rabotnicami (Der Angriff auf den Betrieb durch die Arbeiterinnen) von Kasatkin und Razgon rabočej demonstracii (Die Zerstreuung einer Arbeiterdemonstration) von Viktor Viken'tevič Mazurovskij (1859-1944), ebenfalls aus dem Jahr 19o6, die Brutalität der Ereignisse. ${ }^{79}$ Damit unterscheiden sich die Künstler von ihren westeuropäischen Kollegen, denn bei jenen waren die Szenen des Arbeitskampfes in dieser Zeit überwiegend friedlich. ${ }^{80}$ In allen drei genannten russischen Gemälden bilden die Fabrikgebäude den Hintergrund der abgebildeten Szenen. Sie verweisen damit auf den Ort, an dem die Menschen ihre Unzufriedenheit gemeinsam formulierten und ihren Protest organisierten. Bis zur Oktoberrevolution gab es so gut wie keine russischen Darstellungen, die Streikende innerhalb von Werkshallen und auf dem Fabrikgelände zeigten.

Anfang des 20. Jahrhunderts waren in den urbanen Zentren des Zarenreichs die Industrialisierung und ihre Auswirkungen wie elektrische Beleuchtung oder Straßenbahnen nicht mehr zu übersehen. Die Künstler der Avantgarde nahmen sich in den 1910er Jahren dieser neuen Eindrücke an und verarbeiteten sie in ihren Bildern. ${ }^{81}$ Beispielsweise griffen Natalija Sergeevna Gončarova

78 Boris Michajlovič Kustodiev: Demonstration, 1905. V. Mel'nikova; N. Plavinskaja; E. Volkov: Revoljucija 1905 goda v izobrazitel'nom iskusstve, Moskva 1975, S. 16.

79 V. V. Šleev: Revoljucija $1905^{-1907}$ godov i izobrazitel'noe iskusstvo. Serija al'bomov pod naučnoj redakciej, Bd. 1, Moskva 1977, S. 94; V. V. Šleev: Revoljucija 1905-1907 godov i izobrazitel'noe iskusstvo. Serija al'bomov pod naučnoj redakciej, Bd. 2, Moskva 1977, S. 30-31.

8o Türk: Bilder der Arbeit, S. 212-213.

81 Bowlt: Moskau und St. Petersburg, S. 114-116. 
(1881-1962) und Ol'ga Vladimirovna Rozanova (1886-1918) in ihren Bildern Maschinen, Schornsteine und Fabrikgebäude als Motive auf. Die Künstlerinnen zerlegten die Motive in geometrische Formen und komponierten daraus neue, dynamische Darstellungen. ${ }^{82}$ Bei der Wiedergabe von Bewegung spalteten sie diese in Einzelbilder auf und komponierten daraus innovative Sujets. ${ }^{83}$ Sie begründeten damit einen neuen Umgang mit der industrialisierten Stadt und positionierten sich weder als deren Befürworter noch Kritiker. Marina Ivanovna Cvetaeva (1892-1941) schrieb 1921, dass Gončarova die erste gewesen sei, die Maschinen in ihre Malerei aufgenommen habe, dass dies aber nicht geschehen sei, um den Fortschritt zu feiern. Stattdessen habe die Künstlerin eine eher abwartende Haltung vertreten. ${ }^{84}$ Auch bei Gončarovas Künstlerkollegen lässt sich eine Faszination für die neuen Formen und die Zeichen des Fortschritts beobachten, auch wenn einige der Entwicklung skeptisch gegenüberstanden. ${ }^{85}$

Zur gleichen Zeit entwickelten sich in Westeuropa zwei unterschiedliche Umgangsweisen mit Fabriken. Erstens bildeten neben den Impressionisten auch die Expressionisten Fabriken ab. Unter anderen griffen August Macke (1887-1914), Alexej von Javlensky (Alexej Georgievič Javlenskij, 1864-1941) oder Marianne von Werefkin (Marianna Vladimirovna Verëvkina, 1860-1938) Fabriken in ihren Landschaftsdarstellungen auf. ${ }^{86}$ Allerdings scheint für sie die Faszination von der Form und Ästhetik der Gebäude speziell von der der Schornsteine ausgegangen zu sein. Diese verwendeten sie als geometrische sowie farbliche Elemente. Insgesamt wirken ihre Bilder deutlich ruhiger als diejenigen Gončarovas und Rozanovas, bei denen die Beschleunigung des Alltags für den Betrachter fast körperlich spürbar wird. Zweitens entwickelte sich

82 Beispielsweise die Werke: Natalija Sergeevna Gončarova: Fabrika, 1912; dies.: Dinamomašina, 1913; Olga Vladimirovna Rozanova: Zavod i most, 1913; dies.: Gorod, 1914. Nina Gur'janova: Ol'ga Rozanova i rannij russkij avangard, Moskva 2002, S. 56-57; Helsingin kaupungin taidemuseo (Hrsg.): Olga Rozanova 1886-1918, Helsinki 1992, S. 10.

83 Hierübten diefotografischen Experimente zurWiedergabevon Bewegung entscheidenden Einfluss auf das künstlerische Schaffen der Mitglieder der russischen Avantgarde aus. Barbara Segelken: Energie, Kraft, Geschwindigkeit. Technik und Avantgarde 1910-1925, in: Sabine Beneke; Hans Ottomeyer (Hrsg.): Die zweite Schöpfung. Bilder der industriellen Welt vom 18. Jahrhundert bis in die Gegenwart, Wolfratshausen 2002, S. 266.

84 Hubertus Gassner: „... She Hears the Clock's Galloping Horse inside Herself“, in: Palace Editions Europa; Royal Academy of Arts, London (Hrsg.): From Russia. French and Russian Master Paintings, 1870-1925, from Moscow and St. Petersburg, London 2008, S. $178-187$, S. 184 .

85 Bowlt: Moskau und St. Petersburg, S. 103-104.

86 Beispielsewise: Alexej von Javlensky: Oberau Fabrik, 1910; ders.: Fabrik in den Bergen, 1912; August Macke: Fabrik (Ziegelei), 1912; ders.: Landschaft mit Fabrik, 1913; Marianne von Werefkin: Die Fabrik, 1910/11; dies.: Eisengießerei in Oberstdorf, 1912. 
gerade in Deutschland das Industriegemälde zu einem neuen Repräsentationsmedium. ${ }^{87}$ Die Gemälde feierten die Industrie in einem dokumentarischen, realistischen Stil. ${ }^{88}$ Diese Darstellungen übernahmen vielerorts die Funktion, welche vorher Historiengemälde hatten - Stärke und Ruhm einer Nation darzustellen. Solche Industriegemälde fehlen im Zarenreich völlig.

\subsection{Fazit}

Fabriken waren in der russischen Malerei lange Zeit kaum sichtbar. Weder ließen sich die neuen Bauwerke und Anlagen in russische Landschaften integrieren, noch galten die Arbeiter als Teil des Volkes, dessen Leben die Maler faszinierte. Die russischen Maler des Biedermeiers und die Wanderer interessierten sich eher für Handwerker oder für die ländliche Bevölkerung. Im 19. Jahrhundert hatten Fabriken ihren Platz vornehmlich im Bereich der Druckgrafiken. Die Künstler griffen für ihre Abbildungen Entwicklungen aus Westeuropa auf und übertrugen neuen Darstellungsformen auf russische Motive, wobei lange eine vornehmlich positive Sicht auf Fabriken und Industrie überwog. Neben ausländischen Werken waren es vermutlich in erster Linie diese Abbildungen, mit denen Fotografen in Berührung kamen und die sich auf die Schulung ihres ästhetischen Empfindens auswirkten. Erst um die Jahrhundertwende und besonders im Zuge der revolutionären Ereignisse des Jahres 1905 griffen russische Künstler Fabriken in ihren Gemälden auf und brachten in ihren Bildern Kritik am Umgang mit den einfachen Arbeitern zum Ausdruck. Nur wenige Jahre später entwickelten die Vertreter der russischen Avantgarde eine völlig neue Formensprache, mit der sie der Bewegung der Maschinen, der Geschwindigkeit und der Dynamik der industriellen Entwicklung Ausdruck verliehen. Diese neue Ästhetik war nicht nur im Zarenreich künstlerisch revolutionär, sondern gab auch der ganzen europäischen Kunst neue Impulse.

87 Im Jahr 1912 öffneten im deutschen Kaiserreich zwei Ausstellungen zu Industriebildern ihre Türen. Die Galerie Arnold in Dresden zeigte „Stätten der Arbeit“ und im Sommer 1912 organisiert die Firma Krupp die Ausstellung „Die Industrie in der Bildenden Kunst“. Brandt: Schaffende Arbeit und Bildende Kunst, S. 331.

88 Türk: Bilder der Arbeit, S. 221-222, 226; Walter Fenn: Künstlertum, in: Hermann Glaser; Wolfgang Ruppert; Norbert Neudecker (Hrsg.): Industriekultur in Nürnberg. Eine deutsche Stadt im Maschinenzeitalter, München 1980, S. 275-285, S. 279. 